\title{
Experimental Simulation and Verification of Position Servo Control of Mechanical Rodless Cylinder
}

\section{Yeming ZHANG， Kaimin LI， Hongwei YUE， Shuangyang HE， Dongyuan LI， Kun LYU， Feng WEI}

School of Mechanical and Power Engineering, Henan Polytechnic University, Jiaozuo, China

*Corresponding Author: Yeming ZHANG, E-mail: tazhangyeming@163.com

\begin{abstract}
:
In order to improve the position control accuracy of rodless cylinder, the valve control cylinder system based on pneumatic proportional servo is studied deeply. According to the working principle of the mechanical rodless cylinder control system, under the condition of uniform speed, the driving voltage of the proportional valve is changed to measure multiple sets of friction force and corresponding velocity data. Analyzed the physical structure of each component in pneumatic system, established the mathematical model of pneumatic system, and introduced MATLAB system identification toolbox to identify the parameters of the transfer function. and the experiment verifies its correctness.

Keywords: mechanical rodless cylinder; friction characteristics; mathematical model; parameter identification
\end{abstract}

\section{Introduction}

Pneumatic technology is the technology that uses compressed air as a working medium for energy and signal transmission. It is an important means to improve production efficiency and realize automation of production process in modern industry ${ }^{[1-3]}$. With the continuous progress and development of science and technology and industrial engineering, the requirements of precision operation in industrial occasions are becoming higher and higher, and the precise control of pneumatic technology is put forward higher requirements. In order to realize the accurate control of pneumatic system, it is necessary to analyze the system comprehensively. In the pneumatic valve control cylinder system, the linear model can control the mass flow equation of the valve according to the proportional direction, the mass flow continuity equation of the cylinder, and the dynamics of the cylinder. equation linearization treatment obtained ${ }^{[4-6]}$.

The Pedro Luis Andrighetto ${ }^{[7]}$ Lee E.Schroeder $1^{[8]}$, Brazil in the United States and the laboratory of Beijing Institute of Technology ${ }^{[9]}$ used the test scheme of driving the load cylinder motion directly with compressed air. Zhang Baihai of Beijing Institute of Technology ${ }^{[10]}$ studied the effect of temperature on cylinder friction force, and its experimental results show that the maximum static friction force, viscosity the friction coefficient decreases with the increase of temperature, while the coulomb friction force is less affected by temperature. On the basis of experiments, T. Raparelli and G. Belfore et al. of Italy used the finite element method to analyze the friction force. Through the simulation of the force and deformation of the sealing ring and the verification of the experimental results, the changes of the force and friction force of the double $\mathrm{O}$ seal ring and the lip seal ring under different lubrication and working pressure conditions were studied ${ }^{[11-14]}$.

Liu Xiangming et al . ${ }^{[15]}$ of Wuhan University of Engineering identified the third order electro-hydraulic servo system model by using the $M$ sequence of DAQ acquisition assistant as the excitation signal of the system in LabView environment. song tao et al. ${ }^{[16]}$ of nanjing university of technology established a symmetrical cylinder hydraulic system model. the input and output data of the controlled object of the system were simulated in the MATLAB. the identification model of least square method was used to identify the system, which provided a more accurate system model for further research. Kong Xiangzhen ${ }^{[17]}$ of Shandong University adopted the optimal design problem in the identification process, A pseudo random sequence similar to white noise is used as input signal to continuously excite the controlled object, and the whole system is identified. The input and output experimental data are identified by MATLAB the system identification toolbox. Finally, the transfer function of the whole closed-loop system is obtained. In industrial control, the theory of system identification has been developed 
for decades, and the research and application are more and more extensive, but the research and application in pneumatic servo control need to be further excavated.

Based on the friction experiment, the Stribeck friction model of the rodless cylinder is established. The mass flow equation of proportional valve, the mass flow equation of cylinder and the dynamic equation are established and linearized in the middle position of cylinder. Based on the experimental test, the input and output data of valve control cylinder are obtained, and the parameters of the transfer function obtained are identified in the MATLAB system identification toolbox.

\section{Construction of Control System}

The working principle of pneumatic servo position control system with mechanical rodless cylinder as control object is shown in figure 1. The industrial control computer sends out the target displacement instruction, which converts the numerical signal to the analog voltage signal of $0-10 \mathrm{~V}$ through the D/A conversion module, and the analog voltage signal controls the valve opening in the proportional direction. A measuring system detects the displacement of the rodless cylinder in real time and transmits the displacement signal to the data acquisition card of the industrial control computer in the form of TTL level. The air compressor produces compressed air as a source of fluid power, and flows through pneumatic triplets (air filters, pressure relief valves, oil mist) to transmit clean compressed air into proportional control valves Inside. By using the feedback deviation control, the IPC processes the feedback detection signal and the deviation value of the target value of the displacement measurement system according to the software platform LabVIEW as the upper computer to adjust the valve opening size of the proportional direction control valve in real time, and then reduce the displacement deviation of the rodless cylinder, so that the movement of the rodless cylinder finally reaches the ideal target position. Through the above principle, the pneumatic proportional servo control system can control the precise position of the mechanical rodless cylinder.

According to the experimental principle, set up the following physical experimental platform, as shown in figure 2 .

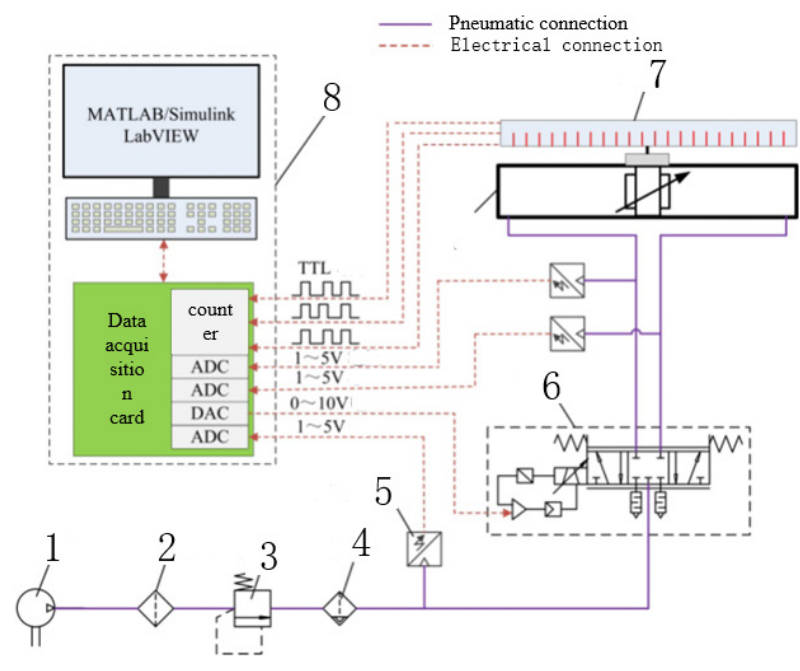

Figure 1 Schematic diagram of pneumatic servo position control system

(1-air compressor; 2-air filter; 3-air regulator; 4-air lubricator; 5-pressure transmitter; 6-proportional directional control valve; 7-Rodless cylinder; 8-IPC).

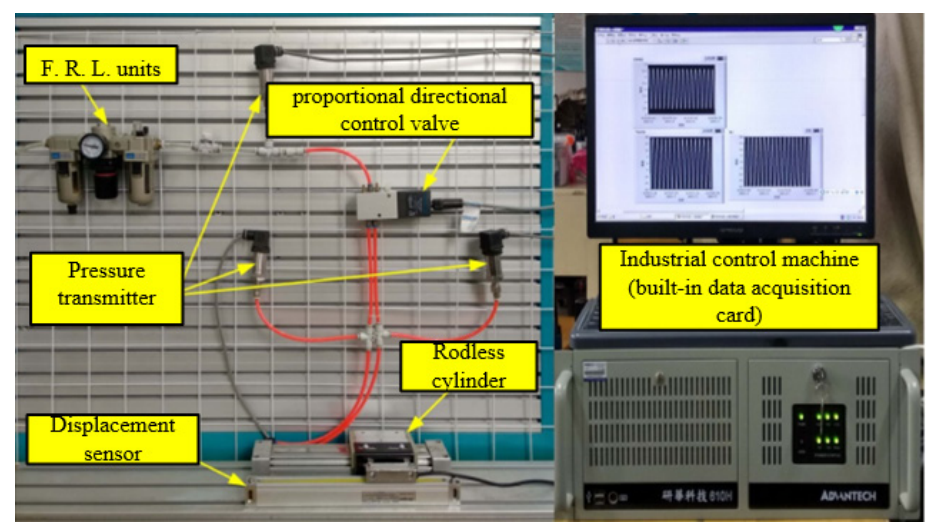

Figure 2 Experimental Platform of Servo Position Control System 
This system proportional direction control valve selects the three-bit five-way proportional direction control valve produced by Germany FESTO Company as the control element, the power supply is DC $24 \mathrm{~V}$, the pressure use range is $0 \sim 1 \mathrm{MPa}$, take the mechanical rodless cylinder as the end pneumatic actuator of the system. In order to ensure the accuracy of measurement and convenient installation, the displacement feedback element selected by this select the grating ruler line displacement sensor.
Checking the displacement characteristics of the Slide valve requires measuring the displacement of the Side valve of the proportional control valve with the change of the driving voltage. Select the HG-C1050 model laser displacement sensor produced by Panasonic Japan is a measuring element. A pressure transducer of MIK-P300 type is selected as the measuring element. The models and parameters of each component are shown in Table 1.

Table 1 Types and working parameters of system components

\begin{tabular}{lll}
\hline \multicolumn{1}{c}{ Components } & \multicolumn{1}{c}{ Model } & \multicolumn{1}{c}{ Parameter } \\
\hline Air compressor & $\begin{array}{l}\text { Medical Silent Piston Air } \\
\text { Compressor } \\
\text { AC3000-03 }\end{array}$ & Working pressure0.4 0.7MPa \\
F. R. L. units & Voltage regulation range0-1MPa \\
proportional directional control valve & MPYE-5-M5-010-B & 5-bit 3-way valve, 0-10V driving voltage \\
Mechanical rodless cylinder & SMC MYC25G-110L & Travel itinerary110mm,Pressure range0.1-0.8MPa \\
Grating Displacement Sensor & JCXE-DC & Operating voltage5V,Travel itinerary 200mm \\
Laser displacement sensor & Panasonic HG-C1050 & Scope of measurement $\pm 15 \mathrm{~mm}$, Precision 30 $\mu \mathrm{m}$ \\
Pressure transmitter & MIK-P300 & Range0-1.0MPa, Precision0.3\%F.S. \\
Flow sensor & SFAB-200U-HQ8-2SV-M12 & Range0-200L/min,Voltage voltage24V \\
\hline
\end{tabular}

\section{Friction characteristics of rodless cylinder}

\subsection{Measurement method of friction parameters}

In the servo system, the friction force always exists in the whole motion process. Compared with the driving force of compressed air, the friction force of pneumatic end actuator is relatively large, and the friction force presents nonlinearly in motion, which seriously affects the accurate positioning of cylinder position. The friction of the cylinder mainly exists in the friction between the piston and the inner wall of the cylinder, between the piston and the inner sealing belt, and between the sliding block and the external dustproof sealing belt. When the rodless cylinder moves in a straight line at a constant speed, the acceleration is zero, and the driving force only works against friction. According to Newton's first law of motion, its mathematical expression is as follows:

$F_{\mathrm{f}}=A_{\mathrm{a}} p_{\mathrm{a}}-A_{\mathrm{b}} p_{\mathrm{b}}$

Therefore, when the cylinder speed is constant, the pressure difference between the two chambers is the friction value to be measured. Under the condition of constant supply gas pressure, by changing the driving voltage of proportional valve to change the opening of valve port, the movement speed of piston will also change. In the experiment, the friction force at different speeds was measured, and the friction-velocity curve was obtained. In the Stribeck friction model, there is the maximum static friction force $F_{s}$, Coulomb friction $F_{c}$, Critical Stribeck velocity $\dot{y}_{\mathrm{s}}$ And viscous friction coefficient $\sigma$ A total of four parameters need to be measured ${ }^{[53]}$. The measurement method is as follows:

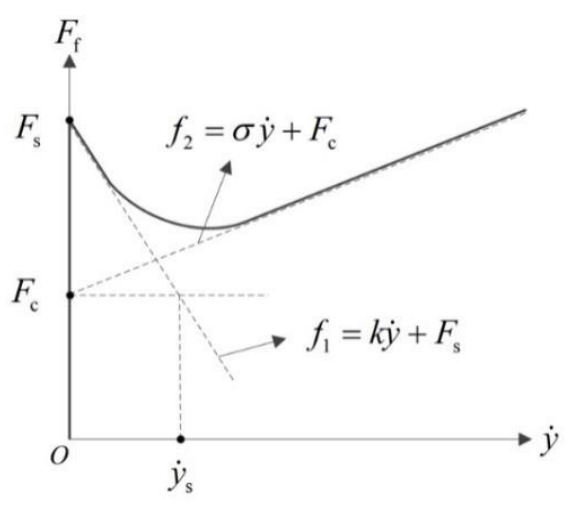

Figure 3 Stribeck friction model curve

In figure 3 , in order to describe the state of friction model intuitively, auxiliary dotted lines are added. When the velocity of the piston is in the zero speed region, the total friction force is $F_{\mathrm{s}}$. With the increase of the speed, the cylinder movement in the low speed stage can be approximately linear $f_{1}$ express. Let $k$ is the slope of this line, and the intersection of the line and the ordinate axis is $F_{\text {s }}$. As the speed continues to increase. It is represented by a straight line $\sigma$, the slope is $\sigma$, the intersection point with the vertical axis is $F_{c}$. Cross the Coulomb friction $f_{2}$ coordinates point as the intersection point of the parallel line of the abscissa axis and the curve $f_{2}$, and then make a vertical line to the abscissa axis, the critical Stribeck velocity is the intersection of the vertical line and the abscissa axis $\dot{y}_{\mathrm{s}}$.

\subsection{Measurement and analysis of friction parameters}

When the supply gas pressure is $0.6 \mathrm{MPa}$, the pressure difference between the two chambers at different constant 
speeds is measured by changing the driving voltage of the proportional directional control valve. The pressure difference of each group under uniform velocity is substituted into the corresponding friction force calculated by formula (1). Figure 4 shows the pressure difference and displacement curve of the cylinder at low speed when the driving voltage is $4.91 \mathrm{~V}$. The maximum static friction force can be obtained by substituting the maximum pressure difference into formula (1), and the friction force at this speed can be obtained by substituting the average pressure difference in the dotted box.It can be seen from the displacement curve that there is a small displacement before the continuous sliding of the cylinder, which is often called the displacement before sliding. The measured friction velocity of each group is summed up and fitted to form a relationship curve, as shown in figure 5 . The four parameter values of Stribeck friction model obtained by making auxiliary lines are shown infigure 6 . The specific parameter values are shown in table 2 .

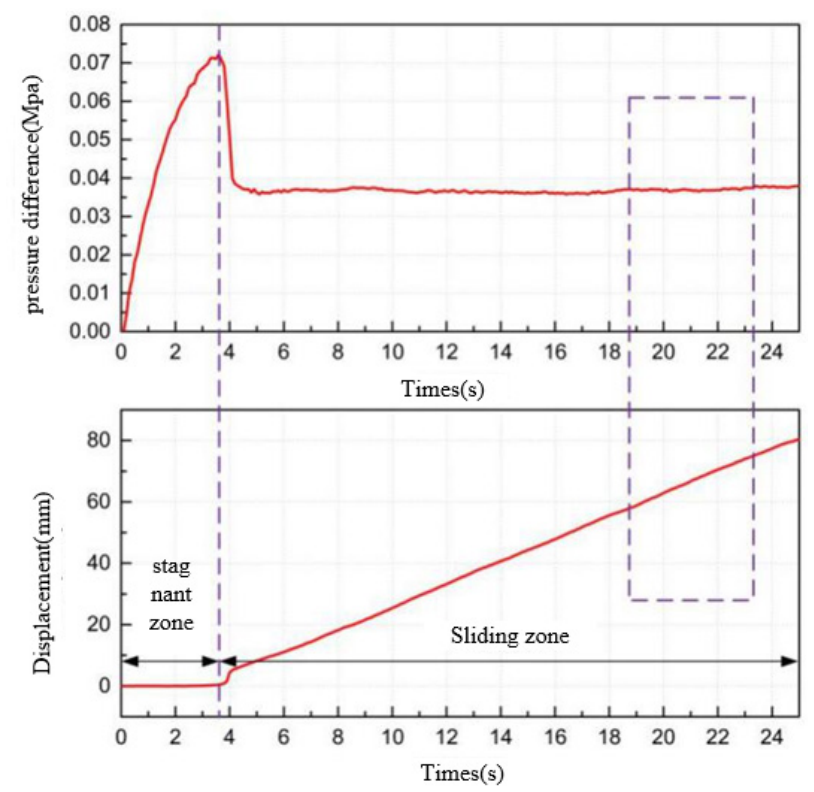

Figure 4 low speed operation process of cylinder

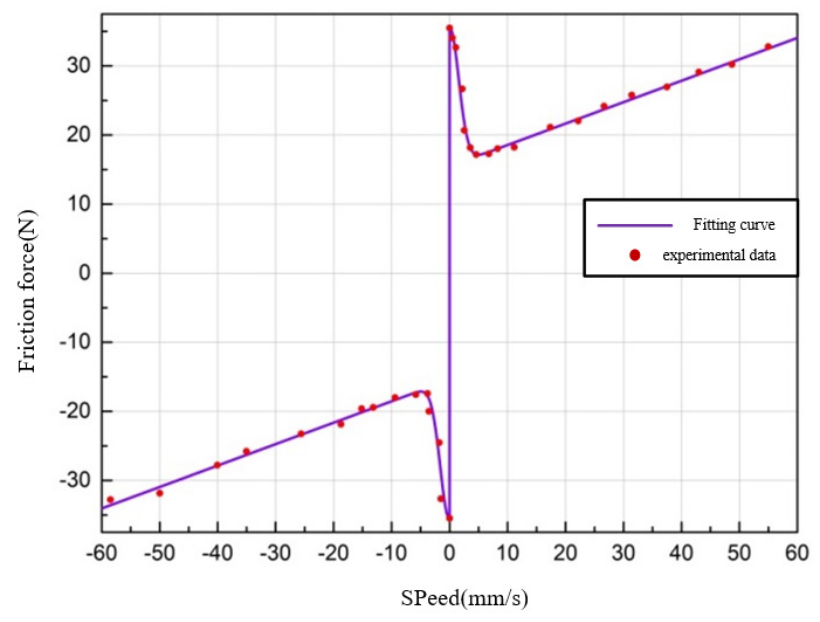

Figure 5 friction curve fitting

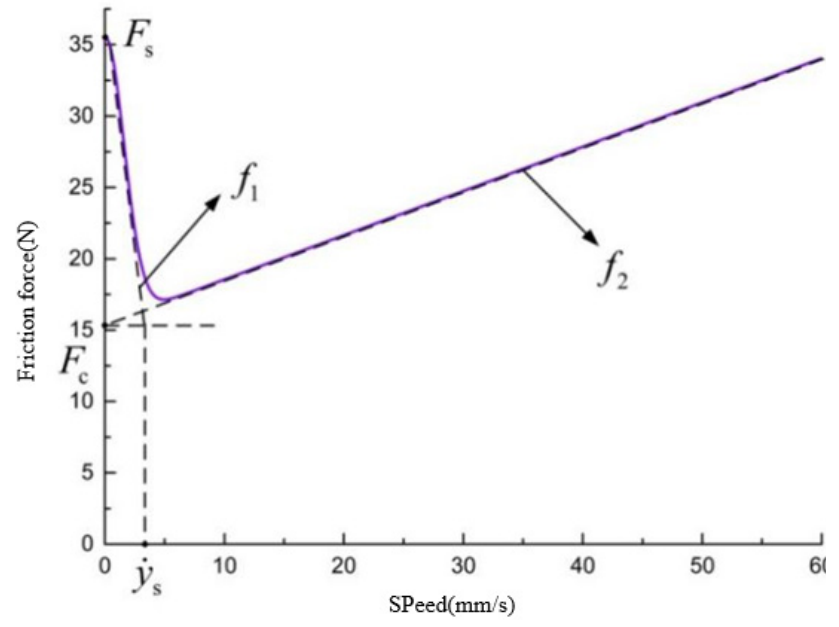

Figure 6 Friction parameter diagram

Table 2 Stribeck friction parameter values

\begin{tabular}{ll}
\hline Parameters & Value \\
\hline$F_{\mathrm{s}}$ & $35.48(\mathrm{~N})$ \\
$F_{\mathrm{c}}$ & $15.31(\mathrm{~N})$ \\
$\dot{y}_{\mathrm{s}}$ & $3.27(\mathrm{~mm} / \mathrm{s})$ \\
$\sigma$ & $0.31(\mathrm{~N} . \mathrm{s} / \mathrm{mm})$ \\
\hline
\end{tabular}

\section{Establishment of system model and parameter identification}

\subsection{Establishment of system model}

By analyzing the physical structure and working principle of proportional directional control valve and rodless cylinder, the piston of rodless cylinder is connected with the external slider of cylinder mechanically, and the displacement of piston is the displacement of slider. The basic equations of valve controlled cylinder system are linearized in the middle position, and the mass flow equation of proportional valve, mass flow continuity equation of rodless cylinder and dynamic equation of are established respectively. Figure 7 describes the flow mechanism of the proportional valve controlled cylinder system. The left chamber of the proportional valve is connected with the chamber a of the cylinder, and the right chamber of the proportional valve is connected with the chamber B of the cylinder. Therefore, any chamber in the proportional valve and the corresponding connected cylinder chamber are regarded as a control body where the gas temperature and pressure are equal everywhere. The control body where the left chamber of the cylinder is located is chamber a, and the control body where the right chamber of the gas cylinder is located is chamber B. the gas flow process in the whole valve controlled cylinder system can be seen It is the process that the gas flows through the proportional valve to charge and deflate a and $\mathrm{B}$ cavities. Set the proportional spool slide to move to the right as a positive direction. When the valve core of the proportional directional valve moves in the positive direction, chamber 
a is filled with air and cavity B is deflated, and the cylinder piston moves to the right.

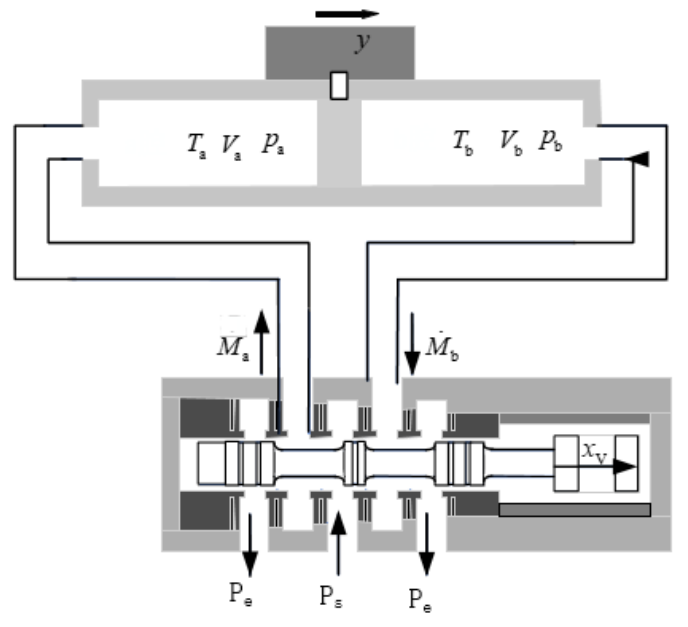

Figure 7 Flow mechanism of valve control cylinder

In figure $7, p_{\mathrm{s}}$ indicates supply gas pressure, $\dot{M}_{\mathrm{a}}$ and $\dot{M}_{\mathrm{b}}$ indicates mass flow in A and B chambers respectively, $p_{\mathrm{a}}$ and $p_{\mathrm{b}}$ indicates the pressure in $\mathrm{A}$ and $\mathrm{B}$ chambers respectively, $T_{\mathrm{a}}$ and $T_{\mathrm{b}}$ indicates the temperature of $\mathrm{A}$ and B cavity respectively, $V_{\mathrm{a}}$ and $V_{\mathrm{b}}$ indicates the volume of A and $\mathrm{B}$ cavity respectively, $x_{\mathrm{v}}$ represents proportional spool displacement, $y$ indicates cylinder piston displacement.

\subsubsection{Proportional Valve Mass Flow Equation}

Because the valve clearance is very small, the mass flow through the gas flow clearance is very small in actual application, so it can be ignored in establishing the model. It is assumed that the working medium is ideal gas satisfying the ideal gas state equation and the gas flow process is isentropic adiabatic process, neglecting the leakage of clearance between spool and sleeve. When the spool moves to the right, the gas in the a-chamber pushes the piston to the right and the piston drives the slider to move synchronously. Figure 8 shows the relative position of the circular orifice to the proportional shoulder.

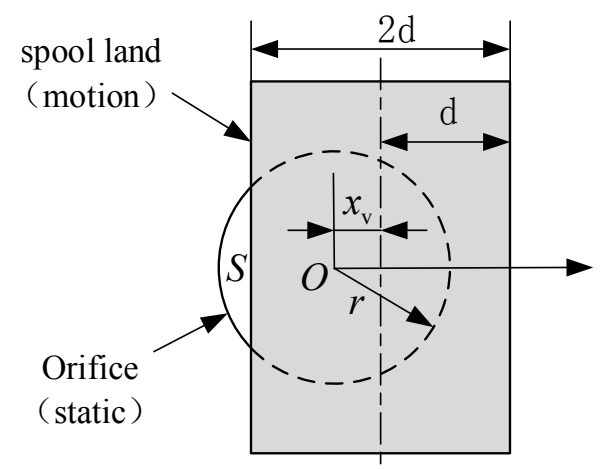

Figure 8 Relative motion position of the shoulder of proportional valve element and the orifice

In figure $8, r$ indicates the radius of the orifice, $2 d$ is the shoulder width of proportional spool, $x_{\mathrm{v}}$ indicates displacement of shoulder relative to orifice, $S$ represents the geometric cross-sectional area of the orifice opening, From the geometric relation in the figure, the expression of the geometric section area $S$ can be obtained as follows:

$S=\left\{\begin{array}{lc}0 & x_{v} \leq d-r \\ r^{2} \arccos \frac{d-x_{v}}{r}-\left(d-x_{v}\right) \sqrt{r^{2}-\left(d-x_{v}\right)^{2}} & d-r<x_{v} \leq d\end{array}\right.$

Based on aerodynamics, considering the shrinkage and friction losses of the orifice, the mass flow through the cylinder a-chamber is as follows ${ }^{[54,55]}$ :

$$
\dot{M}_{\mathrm{a}}= \begin{cases}C S \frac{p_{\mathrm{s}}}{\sqrt{R T_{\mathrm{s}}}} \sqrt{\frac{2 \kappa}{\kappa-1}\left[\left(\frac{p_{\mathrm{a}}}{p_{\mathrm{s}}}\right)^{\frac{2}{\kappa}}-\left(\frac{p_{\mathrm{a}}}{p_{\mathrm{s}}}\right)^{\frac{\kappa+1}{\kappa}}\right]} & \left(\frac{p_{\mathrm{a}}}{p_{\mathrm{s}}} \geq c_{0}\right) \\ C S \frac{p_{\mathrm{s}}}{\sqrt{R T_{\mathrm{s}}}} \sqrt{\frac{2 \kappa}{\kappa+1}\left(\frac{2}{\kappa+1}\right)^{\frac{2}{\kappa-1}}} & \left(\frac{p_{\mathrm{a}}}{p_{\mathrm{s}}}<c_{0}\right)\end{cases}
$$

The mass flow through the b-chamber of the cylinder is:

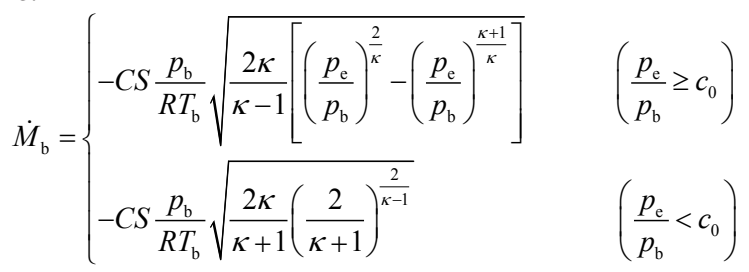

In the formula, $R$ is the gas constant, $C$ is the flow coefficient of the gas through the orifice, $\kappa$ is the isentropic index,critical pressure ratio $c_{0}=\left[\frac{\kappa+1}{2}\right]^{\frac{\kappa}{1-\kappa}}$.

From Taylor's linearization formulas (3) and (4):

$\left\{\begin{array}{l}\Delta \dot{M}_{\mathrm{a}}=c_{1} \Delta x_{\mathrm{v}}-c_{2} \Delta p_{\mathrm{a}} \\ \Delta \dot{M}_{\mathrm{b}}=-c_{1} \Delta x_{\mathrm{v}}-c_{2} \Delta p_{\mathrm{b}}\end{array}\right.$

In the formula, $c_{1}$ represents the mass flow gain of a proportional valve; $c_{2}$ denotes the mass flow pressure coefficient . Since symmetric proportional valves are used, $c$ and $c_{2}$ under initial steady state conditions are represented as follows:

$\left\{\begin{array}{l}c_{1}=\left.\frac{\partial \dot{M}_{\mathrm{a}}}{\partial x_{\mathrm{v}}}\right|_{\substack{p_{\mathrm{a}}=p_{0} \\ x_{\mathrm{v}=0}}} \approx-\left.\frac{\partial \dot{M}_{\mathrm{b}}}{\partial x_{\mathrm{v}}}\right|_{\substack{p_{\mathrm{b}}=p_{0} \\ x_{\mathrm{v}}=0}} \\ c_{2}=-\left.\frac{\partial \dot{M}_{\mathrm{a}}}{\partial p_{\mathrm{a}}}\right|_{\substack{x_{\mathrm{v}=0} \\ p_{\mathrm{a}}=p_{0}}} \approx-\left.\frac{\partial \dot{M}_{\mathrm{b}}}{\partial p_{\mathrm{b}}}\right|_{\substack{x_{v=0}=0 \\ p_{\mathrm{b}}=p_{0}}}\end{array}\right.$

Equation (5) can also be written

$\Delta \dot{M}_{\mathrm{a}}-\Delta \dot{M}_{\mathrm{b}}=2 c_{1} \Delta x_{\mathrm{v}}-c_{2}\left(\Delta p_{\mathrm{a}}-\Delta p_{\mathrm{b}}\right)$

4.1.2 Mass flow equation of cylinder

According to the law of conservation of mass flow, under the isentropic condition, the mass flow of the two chambers in a cylinder is equal to the gas mass in and out of the two chambers in a unit time ${ }^{[56]}$.

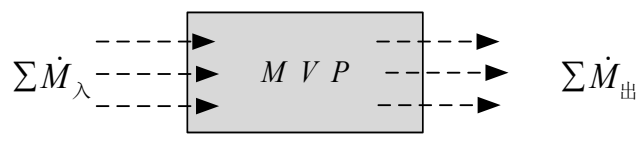

Figure 9 compressed flow into and out of a control body 


$$
\sum \dot{M}_{\lambda}-\sum \dot{M}_{\text {出 }}=\frac{\mathrm{d} M}{\mathrm{~d} t}=\frac{\mathrm{d}(\rho V)}{\mathrm{d} t}=\rho \frac{\mathrm{d} V}{\mathrm{~d} t}+V \frac{\mathrm{d} \rho}{\mathrm{d} t}
$$

The continuous equation for the mass flow of the cylinder cavities $\mathrm{A}$ and $\mathrm{B}$ is as follows:

$$
\left\{\begin{array}{l}
\dot{M}_{\mathrm{a}}=\rho_{\mathrm{a}} \frac{\mathrm{d} V_{\mathrm{a}}}{\mathrm{d} t}+V_{\mathrm{a}} \frac{\mathrm{d} \rho_{\mathrm{a}}}{\mathrm{d} t} \\
\dot{M}_{\mathrm{b}}=\rho_{\mathrm{b}} \frac{\mathrm{d} V_{\mathrm{b}}}{\mathrm{d} t}+V_{\mathrm{b}} \frac{\mathrm{d} \rho_{\mathrm{b}}}{\mathrm{d} t}
\end{array}\right.
$$

The ideal state equation $\rho=p / R T$ of gas is substituted by formula (9) to obtain:

$$
\left\{\begin{array}{l}
\dot{M}_{\mathrm{a}}=\frac{1}{R T_{\mathrm{a}}}\left(p_{\mathrm{a}} \frac{\mathrm{d} V_{\mathrm{a}}}{\mathrm{d} t}+V_{\mathrm{a}} \frac{\mathrm{d} p_{\mathrm{a}}}{\mathrm{d} t}-\frac{p_{\mathrm{a}} V_{\mathrm{a}}}{T_{\mathrm{a}}} \frac{\mathrm{d} T_{\mathrm{a}}}{\mathrm{d} t}\right) \\
\dot{M}_{\mathrm{b}}=\frac{1}{R T_{\mathrm{b}}}\left(p_{\mathrm{b}} \frac{\mathrm{d} V_{\mathrm{b}}}{\mathrm{d} t}+V_{\mathrm{b}} \frac{\mathrm{d} p_{\mathrm{b}}}{\mathrm{d} t}-\frac{p_{\mathrm{b}} V_{\mathrm{b}}}{T_{\mathrm{b}}} \frac{\mathrm{d} T_{\mathrm{b}}}{\mathrm{d} t}\right)
\end{array}\right.
$$

From the isentropic condition:

$$
T=T_{0}\left(\frac{p}{p_{0}}\right)^{\frac{\kappa-1}{\kappa}}
$$

In the formula, $T_{0}$ represents the initial temperature, $p_{0}$ represents initial pressure.Formula (12) can be derived from time by formula (11):

$$
\frac{\mathrm{d} T}{\mathrm{~d} t}=\frac{\kappa-1}{\kappa} \frac{T_{0}}{p_{0}}\left(\frac{p}{p_{0}}\right)^{-\frac{1}{\kappa}} \frac{\mathrm{d} p}{\mathrm{~d} t}=\frac{\kappa-1}{\kappa} \frac{T_{0}}{p}\left(\frac{p}{p_{0}}\right)^{\frac{\kappa-1}{\kappa}} \frac{\mathrm{d} p}{\mathrm{~d} t}=\frac{\kappa-1}{\kappa} \frac{T}{p} \frac{\mathrm{d} p}{\mathrm{~d} t}
$$

Formula (12) can be obtained by substituting it for formula (10):

$$
\left\{\begin{array}{l}
\dot{M}_{\mathrm{a}}=\frac{1}{R T_{\mathrm{a}} \kappa}\left(V_{\mathrm{a}} \frac{\mathrm{d} p_{\mathrm{a}}}{\mathrm{d} t}+\kappa p_{\mathrm{a}} \frac{\mathrm{d} V_{\mathrm{a}}}{\mathrm{d} t}\right) \\
\dot{M}_{\mathrm{b}}=\frac{1}{R T_{\mathrm{b}} \kappa}\left(V_{\mathrm{b}} \frac{\mathrm{d} p_{\mathrm{b}}}{\mathrm{d} t}+\kappa p_{\mathrm{b}} \frac{\mathrm{d} V_{\mathrm{b}}}{\mathrm{d} t}\right)
\end{array}\right.
$$

Because the piston position in the middle of the cylinder is representative of the dynamic characteristics ${ }^{[57]}$, assuming that the parameter of the cylinder piston at the stable working point in the middle is set to the initial value under small interference, the formula (14) can be obtained. The lower corners $0 \mathrm{a}$ and $0 \mathrm{~b}$ indicate the initial state of chamber a and $\mathrm{b}$, respectively, $T_{\mathrm{s}}$ for supply air temperature, $V_{0}$ is half the total cylinder volume, $p_{0}$ is the initial equilibrium pressure of the two chambers when the piston is in steady state.

$$
\begin{cases}\dot{M}_{\mathrm{a}}=0+\Delta \dot{M}_{\mathrm{a}}, & \dot{M}_{\mathrm{b}}=0+\Delta \dot{M}_{\mathrm{b}} \\ p_{\mathrm{a}}=p_{0 \mathrm{a}}+\Delta p_{\mathrm{a}}, & p_{\mathrm{b}}=p_{0 \mathrm{~b}}+\Delta p_{\mathrm{b}} \\ V_{\mathrm{a}}=V_{0 \mathrm{a}}+\Delta V_{\mathrm{a}}, & V_{\mathrm{b}}=V_{0 \mathrm{~b}}+\Delta V_{\mathrm{b}} \\ T_{\mathrm{a}}=T_{0 \mathrm{a}}+\Delta T_{\mathrm{a}}, & V_{\mathrm{b}}=T_{0 \mathrm{~b}}+\Delta T_{\mathrm{b}}\end{cases}
$$

Initial values and have the following relationships:

$$
\left\{\begin{array}{l}
\Delta \dot{V}_{\mathrm{a}}=-\Delta \dot{V}=A \Delta \dot{y} \\
T_{0 \mathrm{a}}=T_{0 \mathrm{~b}}=T_{\mathrm{s}} \\
V_{0 \mathrm{a}}=V_{0 \mathrm{~b}}=V_{0} \\
p_{0 \mathrm{a}}=p_{0 \mathrm{~b}}=p_{0}
\end{array}\right.
$$

Since the incremental values of each physical quantity are small relative to the initial values under small disturbances, they can be neglected in the calculation ${ }^{[58]}$. Therefore, the incremental expression of the continuous equation for the gas mass flow in the cylinder is as follows: $\left\{\begin{array}{l}\Delta \dot{M}_{\mathrm{a}}=\frac{1}{R T_{\mathrm{s}} \kappa}\left[V_{0} \frac{\mathrm{d}\left(\Delta p_{\mathrm{a}}\right)}{\mathrm{d} t}+\kappa p_{0} A \Delta \dot{y}\right] \\ \Delta \dot{M}_{\mathrm{b}}=\frac{1}{R T_{\mathrm{s}} \kappa}\left[V_{0} \frac{\mathrm{d}\left(\Delta p_{\mathrm{b}}\right)}{\mathrm{d} t}-\kappa p_{0} A \Delta \dot{y}\right]\end{array}\right.$

Equation (16) can also be written:

$\Delta \dot{M}_{\mathrm{a}}-\Delta \dot{M}_{\mathrm{b}}=\frac{1}{R T_{\mathrm{s}} \kappa}\left\{V_{0}\left[\frac{\mathrm{d}\left(\Delta p_{\mathrm{a}}\right)}{\mathrm{d} t}-\frac{\mathrm{d}\left(\Delta p_{\mathrm{b}}\right)}{\mathrm{d} t}\right]+2 \kappa p_{0} A \Delta \dot{y}\right\}$

\subsubsection{Dynamic equation of cylinder}

In figure 7, according to Newton's second theorem, the pressure difference between the chambers $\mathrm{a}$ and $\mathrm{B}$ is the power that drives the piston movement. The dynamic equation of the cylinder is as follows:

$$
A\left(p_{\mathrm{a}}-p_{\mathrm{b}}\right)=M_{\mathrm{L}} \ddot{y}+F_{\mathrm{L}}+F_{\mathrm{f}}
$$

Inside, $M_{\mathrm{L}}$ represents the mass of the piston and its moving parts, $F_{\mathrm{L}}$ is the external load force, $F_{f}$ represents the total friction force in motion.

4.1.4 Block diagram and transfer function of valve controlled cylinder system

The linear model of the whole system can be composed of the mass flow equation of proportional directional control valve (7), the mass flow continuity equation of cylinder (17), and the dynamic equation of cylinder (18).

$$
\left\{\begin{array}{l}
\dot{M}_{\mathrm{a}}-\dot{M}_{\mathrm{b}}=2 c_{1} x_{\mathrm{v}}-c_{2}\left(p_{\mathrm{a}}-p_{\mathrm{b}}\right) \\
\dot{M}_{\mathrm{a}}-\dot{M}_{\mathrm{b}}=\frac{1}{R T_{\mathrm{s}} \kappa}\left[V_{0}\left(\frac{\mathrm{d} p_{\mathrm{a}}}{\mathrm{d} t}-\frac{\mathrm{d} p_{\mathrm{b}}}{\mathrm{d} t}\right)+2 \kappa p_{0} A \dot{y}\right] \\
A\left(p_{\mathrm{a}}-p_{\mathrm{b}}\right)=M_{\mathrm{L}} \ddot{y}+F_{\mathrm{L}}+F_{\mathrm{f}}
\end{array}\right.
$$

Make the pressure difference $p_{\mathrm{L}}=p_{\mathrm{a}}-p_{\mathrm{b}}$, Mass flow difference $\dot{M}_{\mathrm{L}}=\dot{M}_{\mathrm{a}}-\dot{M}_{\mathrm{b}}$, The formula (19) is transformed by pulling:

$$
\left\{\begin{array}{l}
\dot{M}_{\mathrm{L}}(s)=2 c_{1} x_{\mathrm{v}}(s)-c_{2} p_{\mathrm{L}}(s) \\
\dot{M}_{\mathrm{L}}(s)=\frac{1}{R T_{\mathrm{s}} \kappa}\left[V_{0} s p_{\mathrm{L}}(s)+2 \kappa p_{0} A s y(s)\right] \\
A p_{\mathrm{L}}(s)=M_{\mathrm{L}} s^{2} y(s)+F_{\mathrm{L}}(s)+F_{\mathrm{f}}(s)
\end{array}\right.
$$

The block diagram of valve controlled cylinder system can be obtained from formula (20), as shown in figure 10 .

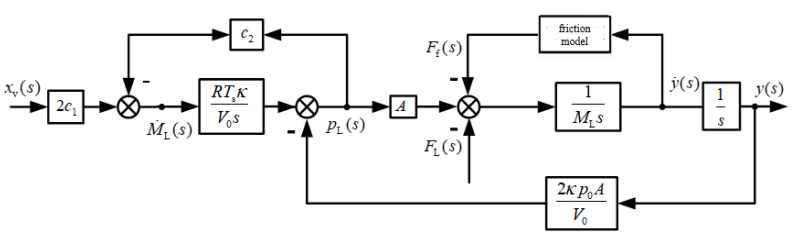

Figure 10 Block diagram of the valve-controlled cylinder system

The transfer function of the valve controlled cylinder system can be obtained by solving the simultaneous basic equations or by simplifying the block diagram. 
(1) Transfer function of spool displacement $x_{v}$ to cylinder output displacement $y$.

When $F_{\mathrm{f}}=0$ and $F_{\mathrm{L}}=0$, the transfer function of spool displacement $x_{\mathrm{v}}$ to output displacement $y$ can be obtained as follows:

$\frac{y_{x}(s)}{x_{\mathrm{v}}(s)}=\frac{K_{\mathrm{T}} \omega_{\mathrm{n}}^{2}}{s\left(s^{2}+2 \zeta \omega_{\mathrm{n}} s+\omega_{\mathrm{n}}^{2}\right)}$

Among, the gain $K_{\mathrm{T}}$ is: $K_{\mathrm{T}}=\frac{c_{1} R T_{\mathrm{s}}}{p_{0} A}$

The damping ratio $\zeta$ is:

$\zeta=\frac{c_{2} R T_{\mathrm{s}}}{2 A} \sqrt{\frac{\kappa M_{\mathrm{L}}}{2 V_{0} p_{0}}}$

The natural frequency $\omega_{\mathrm{n}}$ is:

$\omega_{\mathrm{n}}=\sqrt{\frac{2 \kappa p_{0} A^{2}}{V_{0} M_{\mathrm{L}}}}$

When $x_{\mathrm{v}}=0$ and $F_{\mathrm{L}}=0$, it can be obtained that the transfer function of the friction force $F_{\mathrm{f}}$ to the output displacement $y$ is:

$\frac{y_{\mathrm{f}}(s)}{F_{\mathrm{f}}(s)}=-\frac{\frac{K_{\mathrm{T}} \omega_{\mathrm{n}}^{2}}{2 c_{1} A}\left(\frac{V_{0}}{R T_{\mathrm{s}} \kappa} s+c_{2}\right)}{s\left(s^{2}+2 \zeta \omega_{\mathrm{n}} s+\omega_{\mathrm{n}}^{2}\right)}$

In the same way, when $F_{\mathrm{f}}=0$ and $F_{\mathrm{f}}=0$, it can be obtained that the transfer function of friction force $y$ to output displacement $y$ is:
$\frac{y_{\mathrm{L}}(s)}{F_{\mathrm{L}}(s)}=-\frac{\frac{K_{\mathrm{T}} \omega_{\mathrm{n}}^{2}}{2 c_{1} A}\left(\frac{V_{0}}{R T_{\mathrm{s}} \kappa} s+c_{2}\right)}{s\left(s^{2}+2 \zeta \omega_{\mathrm{n}} s+\omega_{\mathrm{n}}^{2}\right)}$

Therefore, the total transfer function of the valve controlled cylinder system is:

$$
\begin{aligned}
& y(s)=y_{x}(s)+y_{\mathrm{f}}(s)+y_{\mathrm{L}}(s) \\
& =\frac{K_{\mathrm{T}} \omega_{\mathrm{n}}^{2} x_{\mathrm{v}}(s)-\frac{K_{\mathrm{T}} \omega_{\mathrm{n}}^{2}\left[F_{\mathrm{f}}(s)+F_{\mathrm{L}}(s)\right]}{2 c_{1} A}\left(\frac{V_{0}}{R T_{\mathrm{s}} \kappa} s+c_{2}\right)}{s\left(s^{2}+2 \zeta \omega_{\mathrm{n}} s+\omega_{\mathrm{n}}^{2}\right)}
\end{aligned}
$$

Because the proportional directional valve has strong linear characteristics, the transfer function can be described as follows:

$\frac{x_{\mathrm{v}}(s)}{U(s)}=K_{\mathrm{v}}$

In the formula, $K_{\mathrm{v}}$ represents proportional gain, $U$ indicates the drive voltage of the valve.

The transfer function of the proportional amplifier is: $\frac{U(s)}{y_{\text {in }}(s)-y(s)}=K_{\text {a }}$

In the formula, $K_{\mathrm{a}}$ represents proportional gain, $y_{\text {in }}$ represents the input displacement.

Friction compensation is used to reduce the influence of friction on the system. According to the formulas (27), (28), (29), the block diagram of the cylinder servo system can be obtained as shown in figure 11 .

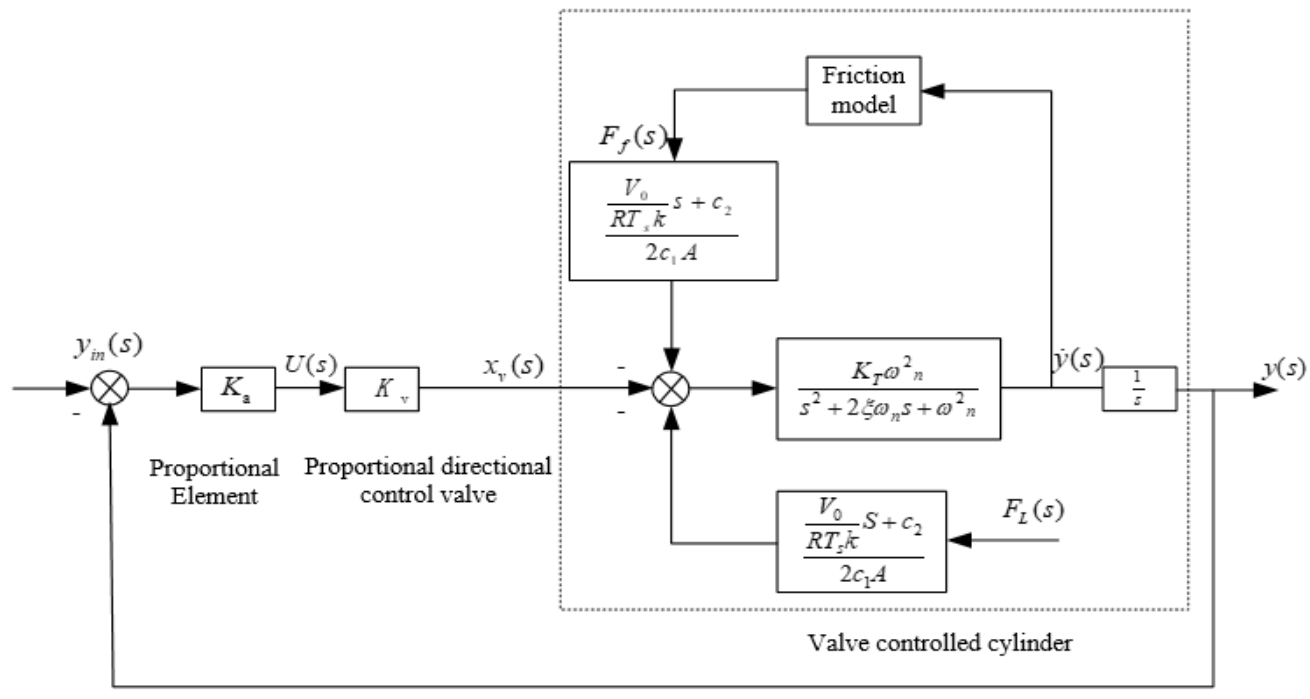

Figure 11 Block Diagram of Cylinder Position Servo System

Therefore, the open-loop transfer function of the system is:

$$
H(z)=\frac{b_{1} z^{3}+b_{2} z^{2}+b_{3} z+b_{4}}{a_{1} z^{3}+a_{2} z^{2}+a_{3} z+a_{4}}
$$

Formula (4-29) is discretized and its open-loop transfer function is obtained as follows:

$$
H(z)=\frac{b_{1} z^{3}+b_{2} z^{2}+b_{3} z+b_{4}}{a_{1} z^{3}+a_{2} z^{2}+a_{3} z+a_{4}}
$$

In the formula, $b_{1}, b_{2}, b_{3}, b_{4}$ and $a_{1}, a_{2}, a_{3}, a_{4}$ are constant values.

\subsection{Valve-controlled cylinder system identification}

\subsubsection{System Identification Process}

System identification can be divided into open loop identification and closed loop identification. Closed-loop identification methods are divided into closed-loop direct identification and closed-loop indirect identification. The closed-loop indirect identification method is to identify the closed-loop transfer function of the whole system first, 
then subtract the feedback link of the system to obtain the open-loop transfer function of the system. This method is cumbersome due to the elimination of intermediate links. Closed-loop direct identification method is to identify the open-loop transfer function of controlled object under closed-loop condition. Since the linearized system model is a third-order model and the proportional valve-controlled cylinder system has an integral part and is an open-loop unstable system, this paper uses closed-loop condition to directly identify the open-loop transfer function of the system.
M-sequence is a binary pseudo-random code sequence of approximate white noise signals as shown in figure 12. M-sequence as interference signal can fully excite the characteristics of the controlled object. The input signal is the superposition of the controller output signal and M-sequence signal, and the output signal is the cylinder displacement signal. To identify the input signal and output displacement before and after the object, the open loop system of the system is identified. Figure 13 is the identification test schematic.

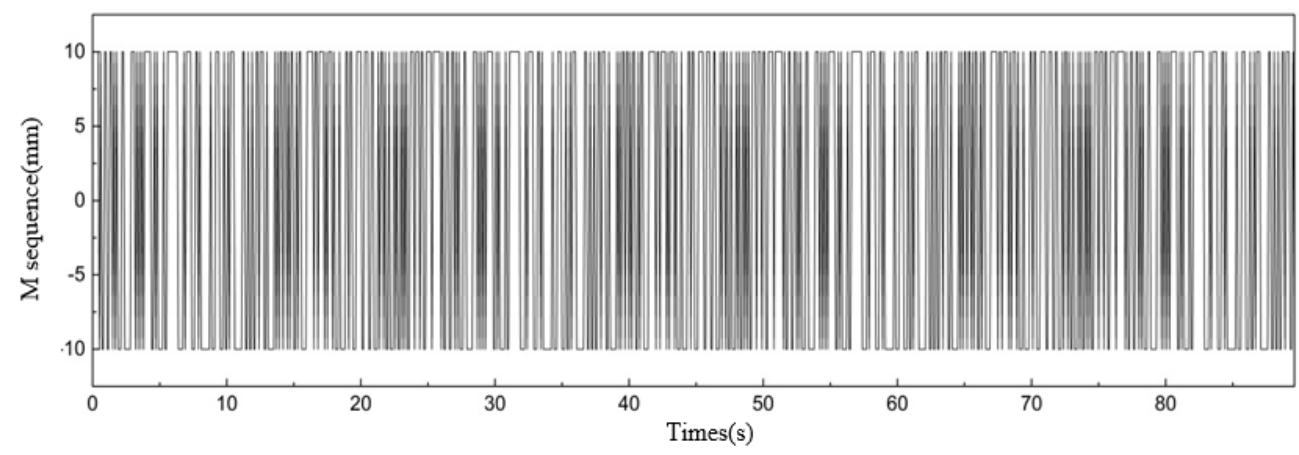

Figure $12 \mathrm{M}$-sequence signal

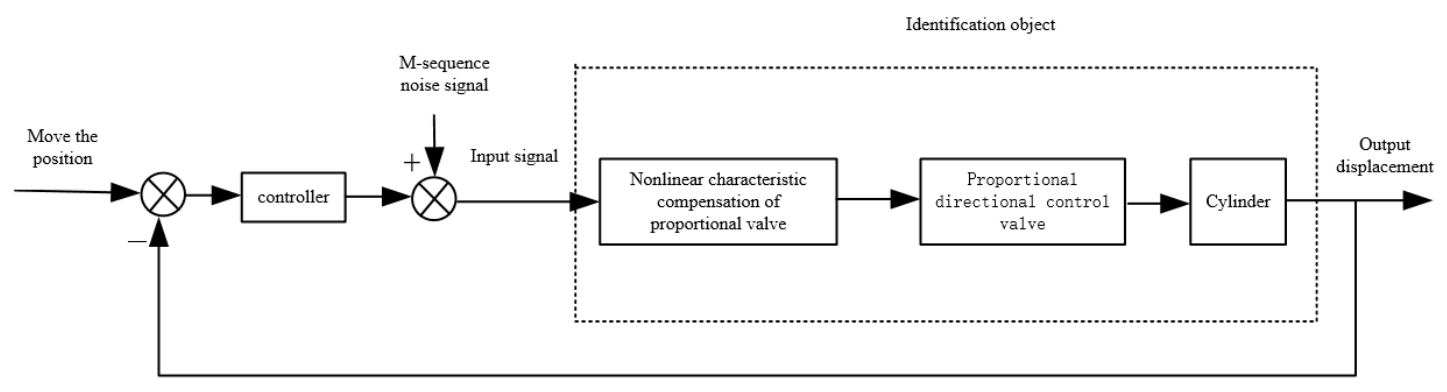

Figure 13 Identification test schematic diagram

For nonlinear systems, a parametric linearization model can be established based on the perturbation theory. During the identification process, the cylinder piston is allowed to move to the left and right, and the "near disturbance" of the input signal to the system should be in both positive and negative directions. The amplitude of input signal is not too small, it will slow down the response of the system, not fully excite the dynamic characteristics of the system and make the system identify distortion, but it should not be too large, so the input signal should be selected reasonably. Identified input and output signals are shown in figure 14.

In MATLAB, input signal and output signal are imported separately. ARX discrete transfer function model is selected for identification in time domain. The identification results are shown in figure 15.

The identified system model is:

$H(z)=\frac{0.1496 z^{2}-0.03607 z+0.1678}{z^{3}-1.4276 z^{2}+0.85537 z-0.4243}$

\subsubsection{Experimental verification of system identification model}

According to the system schematic diagram built as shown in figure 16, the input step signal and sinusoidal displacement signal are experimentally verified. Figure 17 is a comparison diagram of the results of simulation and experiment. It can be concluded that the identified system model can well reflect the dynamic characteristics of the control system.

From the previous section 4.1 (Establishment of system model) the theoretical model of the system is obtained. Under the standard input signal (step signal and sinusoidal signal), the position control of the rodless cylinder is studied experimentally, and the actual input and output data curve of the rodless cylinder is obtained. Based on the input and output data of the rodless cylinder system, the identification model of the rodless cylinder system is obtained by using the MATLAB system identification toolbox. Finally, with the identification model as the system and the standard input signal as the excitation, the experimental curve and the simulation curve are tracked. 

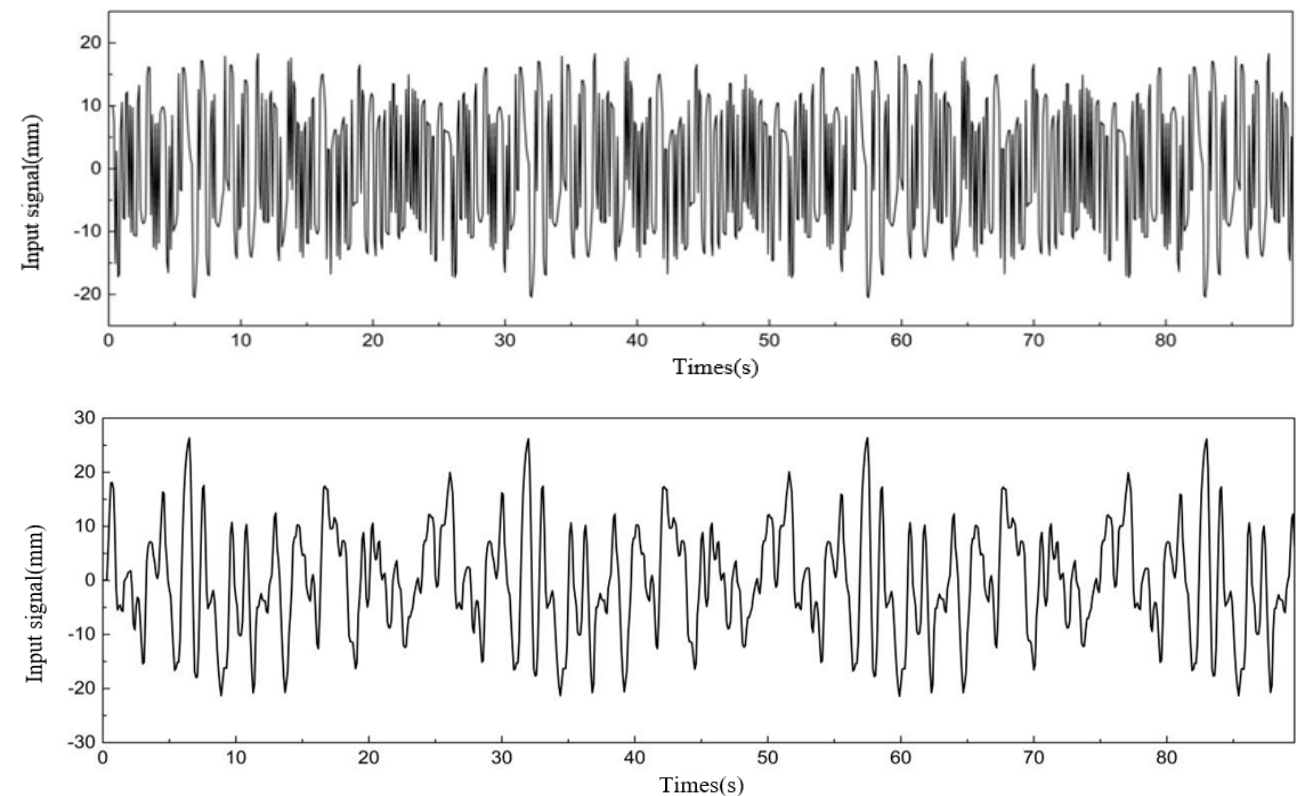

Figure 14 identification of input and output signals

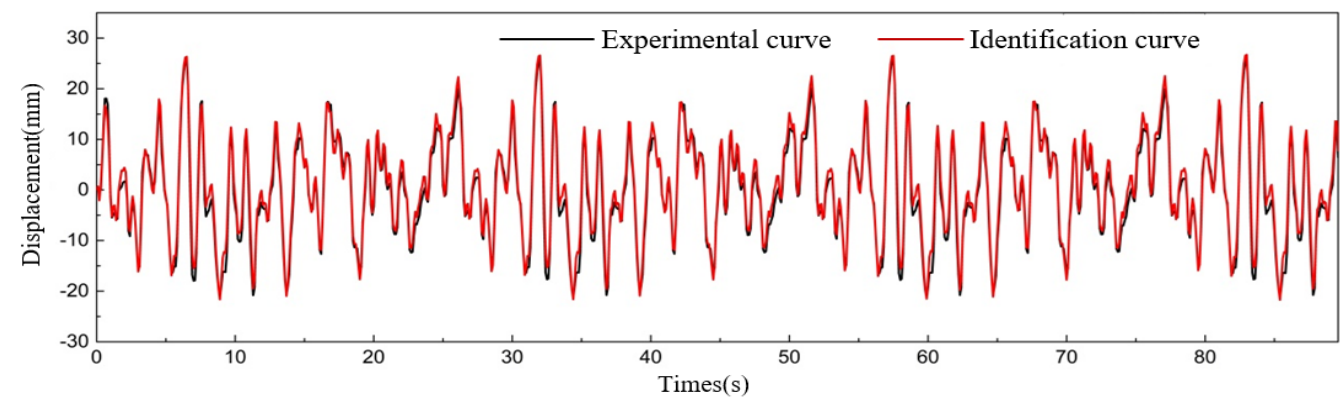

Figure 15 Identification result

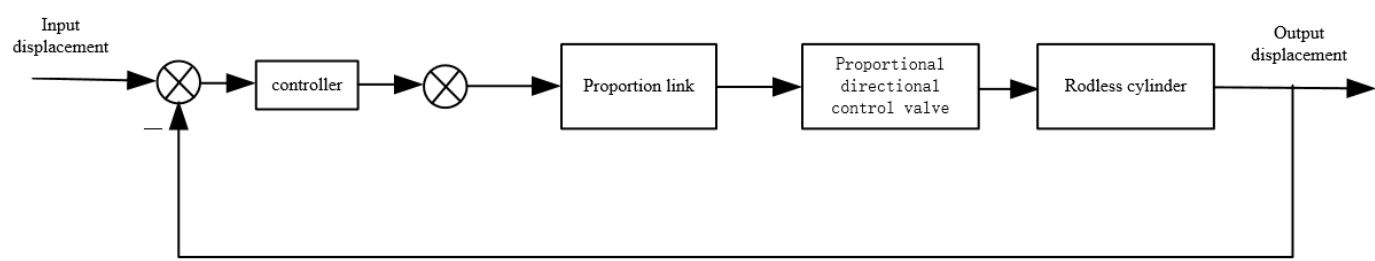

Figure 16 system principle block diagram

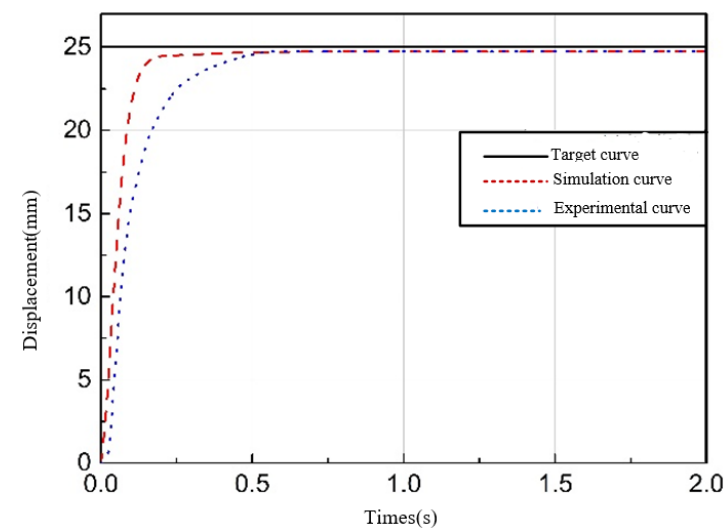

(a) Positioning result;

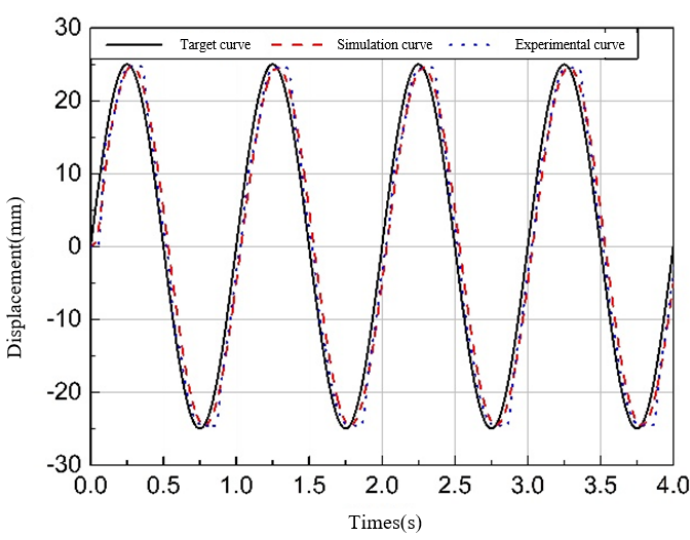

(b) Position tracking result

Figure 17 Comparisons between simulation and experiment 


\section{Conclusions}

Based on the experimental system of position servo control of rodless cylinder, the conclusion is as follows :(1) The friction model of Stribeck mechanical rodless cylinder is established. $\left(F_{\mathrm{s}}=35.48 \mathrm{~N}, \quad F_{\mathrm{c}}=15.31 \mathrm{~N}, \dot{y}_{\mathrm{s}}=3.27 \mathrm{~mm} / \mathrm{s}\right.$, $\sigma=3.27 \mathrm{~mm} / \mathrm{s}$ ) ; (2) By establishing the mass flow equation of proportional control valve, mass flow equation and dynamic equation of cylinder, and reducing the influence of friction on the system by friction compensation, the accurate mathematical model of proportional control cylinder system is finally established .(3) The input and output data of valve control cylinder are obtained by experimental test, and the parameters of the system transfer function obtained by MATLAB system box identification to used to verify the correctness of the system model.

Author Contributions: Conceptualization, Yeming ZHANG and Hongwei YUE; Data curation, Kaimin LI; Funding acquisition, Yeming ZHANG; Project administration, Yeming ZHANG; Software, Shuangyang HE and Dongyuan LI; Supervision, Kun LYU and Feng WEI; Writing-original draft, Kaimin LI and Hongwei YUE; Writing-review \& editing, Yeming ZHANG and Kaimin LI.

Conflict of Interest: The authors declare no conflict of interest.

Funding: The paper is Supported by Henan Province Science and Technology Key Project (Grant No. 202102210081), the Fundamental Research Funds for Henan Province Colleges and Universities (Grant No. NSFRF140120), Doctor Foundation of Henan Polytechnic University (Grant No.B2012-101).

Acknowledgments: The authors would like to thank Henan Polytechnic University and Beihang University for providing the necessary facilities and machinery to build the prototype of the pneumatic servo system. The authors are sincerely grateful to the reviewers for their valuable review comments, which substantially improved the paper.

\section{References}

[1] SMC (China) Co., Ltd. Modern Practical Pneumatic Technology[M]. Beijing: Machinery Industry Press ,2008.

[2] Ming Y. Air compressor efficiency in a Vietnamese enterprise [J]. Energy Policy, 2009, 37(6): 2327-2337.

[3] Zhang Yeming, Cai Maolin. Overall Life Cycle Comprehensive Assessment of Pneumatic and Electric Actuator[J]. Chinese Journal of Mechanical Engineering, 2014, 27(3):584-594.

[4] Zhang Y, Li K, Wei S, et al. Pneumatic Rotary Actuator Position Servo System Based on ADE-PD Control [J].
Applied Sciences, 2018, 8(3): 406.

[5] Yeming Zhang, Ke Li, Geng Wang, Maolin Cai. Nonlinear model establishment and experimental verification of a pneumatic rotary actuator position servo system[J]. Energies 2019, 12(6), 1096.

[6] Yeming Zhang, Hongwei YUE, Ke Li, Maolin Cai. Analysis of power matching on energy saving of pneumatic rotary actuator servo-control system[J]. Chinese Journal of Mechanical Engineering, 2020, 33:30.

[7] Pedro Luís Andrighetto, Antonio Carlos Valdiero, Leonardo Carlotto. Study of the frictionbehavior in industrial pneumatic actuators. ABCM Symposium Series in Mechatronics,2006, 2: 369-376.

[8] Lee E. Schroederl. Experimental Study of Friction in a Pneumatic Actuator at ConstantVelocity. Jourmal of Dynamic Systems, Measurement, and Control, 1993, 9,Vol. 115:575-577.

[9] Zhang Baihai, Cheng Haifeng, Ma Yanfeng. Experimental study of cylinder friction. Beijing Institute of Technology Journal ,2005,(6):483-487.

[10] Zhang Baihai, Peng Guangzheng, Liu Qiong, Study on the Influence of Temperature on Friction Characteristics of Cylinder.

[11] T. Raparelli, A. Manuello, Bertettot and L. Mazzat. Experimental and Numerical Study offriction in an Electrometric Seal for Pneumatic Cylinders. Tribology International. 1997,Volume 30 Number 7: 547-557.

[12] Belforte. G Raparelli. T, Velardocchia. M. Study of the Behavior of Lip Seals in PneumaticActuators.. Lubr. Eng,1993, 45: 775-780.

[13] Conte M, Manuello A, Mazza L, Visconte C. Measurement of contact pressure in pneumaticactuator seals. In: Proceedings of the AITC-AIT 2006 international conference on tribology,2006.

[14] G. Belforte, M. Conte, A. Manuello Bertetto, L. Mazza, C. Visconte. Experimental andnumerical evaluation of contact pressure in pneumatic seals. Tribology International. 2009,42:169- 175.

[15] Liu Xiangming, Li Liang. LabVIEW Electro-hydraulic System Identification [J]. Machine and Hydraulic ,2007,35(9):143-145.

[16] Song Tao, Yu Cungui. Simulation based on MATLAB/ Simulink hydraulic servo system identification [J]. Hydraulic and Pneumatic ,2015,(10):120-123.

[17] Kong Xiangzhen. Dynamic Characteristics and Control of Pneumatic Proportional System [D]. Jinan; Shandong University ,2007. 